\title{
Towards Social Networks Integrated Domain-Specific Business Directories: A Design Science Approach
}

\author{
Atia B. Memon ${ }^{1,}{ }^{*}$, Arifa Bhutto ${ }^{2}$, Kirshan K. Luhana ${ }^{1}$, Altaf H. Abro ${ }^{1}$, Kyrill Meyer ${ }^{3}$ \\ ${ }^{1}$ Department of Computer Science, University of Sindh, Jamshoro, Pakistan. \\ ${ }^{2}$ Institute of Information and Communication Technology, University of Sindh, Jamshoro, Pakistan. \\ ${ }^{3}$ Institute for Digital Technologies, Leipzig, Germany. \\ ${ }^{*}$ Corresponding author: atia.memon@usindh.edu.pk
}

Abstract

This research work relates to the paradigm of business pages hosted on social networking sites, as they are increasingly gaining attention among business organizations as a potential source of their community outreach. However, the social networking sites, whilst offering several necessary community services, are currently lacking the support for the domain specific features of specific types of organizations such as research and innovation laboratories, educational and training institutes and many others. Undertaking a design science research approach, we investigate the stated problem in more detail in a specific type of organizations termed as Innovation Laboratories. As a result, we propose designing domain specific business directories while simultaneously integrating them with the business pages hosted on existing social networking sites. We demonstrate the feasibility and application of our proposed model through a web-based tool named as "SIDIL: Social networks Integrated Directory of Innovation Laboratories". The tool has been evaluated by the experts running Innovation Laboratories and found effective in achieving its objectives. As a conclusion, we present a motivation towards designing next generation of online and integrated business networking platforms.

Keywords-Business pages, business networking platforms, online business directories, social networking sites, social data integration

\section{Introduction}

$\mathrm{N}$ OWADAYS, with the ubiquitous computing and networking resources and increasing tendency towards digitization, traditional paper-based business directories (e.g., white pages, yellow pages, and other industry-specific directories) have turned out to be insufficient and also ineffective for searching and connecting with businesses of interest. With the passage of time, more and more attention is being given to online business directories varyingly termed as internet/digital/online yellow pages, citation sites, online business listings, etc. Getting included in one or more business directories makes all types of businesses more discoverable and reputable among potential customers as well as other business partners. An online business directory, analogous to its paper counterpart, serves

ISSN: 2523-0379 (Online), ISSN: 1605-8607 (Print)

DOI: https://doi.org/10.52584/QRJ.1901.04

This is an open access article published by Quaid-e-Awam University of Engineering Science $\&$ Technology, Nawabshah, Pakistan under CC BY 4.0 International License. as a portal where businesses list themselves and the potential customers search these listings for the qualified vendors of their interest. The directories work in two directions: (i) the customers value these directories for the information, and (ii) businesses value these directories for advertising to customers [1]. The listings are usually categorized among predefined business categories and are built upon NAP (i.e., Name, Address, and Phone number) protocol. Currently, a number of online business directories in different forms exist; both general as well as specialized for different niches, regions, and industries that vary in terms of information and interaction possibilities they offer. On the baseline, there are the directories that allow the listed businesses to merely include their basic information in terms of name, address, and phone number. The expanded form of these directories offer listings also by email, website, fax number, and the like. The advantage of these directories over the traditional printed directories is that they enable businesses to easily 
update their details and thus keep their information current. On the advanced level, there are the directories that in addition to basic profile of listed business also allow the visitors to leave comments for future visitors. The facility of posting comments on the one hand aid the qualified sellers in building reputations with positive remarks and on the other hand assist the motivated buyers to compare, decide, and select businesses accordingly.

While these online directories have somehow overcome the shortcomings of physical business directories, many times while using an online directory a user cannot target a search among thousands of business listings. The directories, as are limited to searching by business category, name, and address only, offer too broad searching and thereby return too many results in response to a search request. They do not facilitate searching by specific attributes such as opening hours, products, specialties, expertise, etc. Also, once a list of potential sellers is found, the user cannot use the online medium to contact the selected business directly. This leaves the user with cumbersome task to contact the potential sellers through telephone number separately. In many cases, user wants to contact multiple sellers and have to contact them sequentially. Besides customers, this is unwanted for sellers as well who want to use same platform for advertising and keeping their customers continuously updated regarding their products and services.

In this realm, a recent and advanced embodiment of online business directories that merge the listing and searching of business pages with the real-time communication and interaction facilities are the business pages hosted on social networking sites [2]. Nowadays, many leading social networking sites including the Facebook, LinkedIn, Google+, and Xing are hosting business pages. Through business pages, social networking sites offer a number of benefits and interaction possibilities that were previously not possible or very difficult to acquire for the listed businesses [3]. On a business page, a business can describe their profile beyond name, address and phone number and may also include products, specialties, email, URL, fax number, multiple locations, map entry, textual description, foundation year, milestones, and other general information. Once a business page is created and made public, the listed business can share its updates, advertise new products/services, solicit customer reviews, collect customer demands, and communicate with customers through public comments on shared updates as well as private one-to-one messages. Many of these platforms also offer audio/video calling mechanism direct from the platform. Additionally, customers can express their acquaintance with the business by liking or following the page and thus get notified when some new content is added to the page by the listed business. Businesses are also served with page analytics to determine their page traffic and activity. Altogether these mechanism have made business pages most wanted and effective medium for B2C as well as B2B connections.

\section{Problem Statement}

One major challenge of the extant forms of business directories, even the advanced manifestations like business pages, is that they all lack support for representation of domain-specific attributes of special type of organizations such as research and innovation laboratories, educational and training institutes, hospitals, to name a few. Furthermore, the architectures of most online directories is framed in a manufacturing firm style, and thus they all fall insufficient when it comes to the representation of organizations where there is no tangible product or service for selling or marketing purposes [4]. In such circumstances, potential customers cannot search and filter results for such type of organizations according to a certain specific criteria and thereby get ambiguous results with incomplete information for decision making. This brings challenges for both. On one hand, the customers face difficulties in locating organizations of their interest, retrieving complete list of potential providers for the service they are searching for, filtering and selecting a particular organization, and thus making informed and effective decisions. On the other hand, the organizations miss the opportunity of reaching to all potential customers and therefore they lose the customers and their business is limited.

\subsection{Research Objectives}

The main question guiding this research work is how we can facilitate special type of organizations in having complete web representation in terms of their general as well as domain specific attributes?. In order to address this research question, the present paper proposes to design domain-specific business directories along with seamless integration of business pages hosted on social networking sites. The proposed design offers the rationale and technological grounds for a new model of next generation of integrated online business directories. Such integrated business directories will enable to achieve two way objectives. One, they will alleviate the lacking of special type of organizations in representing themselves in a dedicated manner on online platforms. The organizations will have online profile similar to how they are described in physical 
environment. This will make them discoverable and interconnected in global business environment. Two, it will enhance the usability and usefulness of phenomenon of social networking site business pages. The special type of organizations will be leveraging and updating their business pages even more as now they will have a chance to bring and integrate same information within other potentially more dedicated and specialized business directories.

In doing so, the problem is investigated and motivated in more details by examining a special type of organizations termed as Innovation Laboratories (section 3). Following this, based on the potential of business pages hosted on social networking sites in facilitating the identified requirements of Innovation Laboratories, we propose the model for integrated design of domain-specific online business directories (section 3). We show the feasibility of our proposed design by developing a case application named as SIDIL - Social networks integrated directory of Innovation Laboratories - that lists Innovation Laboratories in terms of their both the general and domain specific attributes and integrates their information available on their business pages hosted on Facebook, LinkedIn, Google+, and Xing networks (section 4). The SIDIL application has been evaluated by Innovation Laboratory facilitators in terms of its effectiveness and usability and found effective in achieving its stated objectives (section 4).

\section{Research Design}

The work discussed herein is undertaken following a Design Science Research (DSR) approach. DSR aims at addressing real world problems by creating artifacts that combine both novelty and utility [5]. The DSR employed for current study corresponds to the development of a working IT artifact that model how we can supplement special type of organizations in specifying their domain-specific attributes while simultaneously leveraging the potential of existing social networking sites. Academic literature suggests several DSR methods for creating successful artifacts. We build upon two main phases of DSR process drawn upon [6] and [7]: the problem investigation and proposed solution, and the artifact implementation and evaluation. Figure 1 depicts the overall research procedure.

In the first phase, the problem addressed herein was investigated in details through a problem-driven approach [8]. In doing so, the state of the art of a specific type of organizations called Innovation Laboratories (InnoLabs) and their general as well as domainspecific attributes were revealed from the literature.

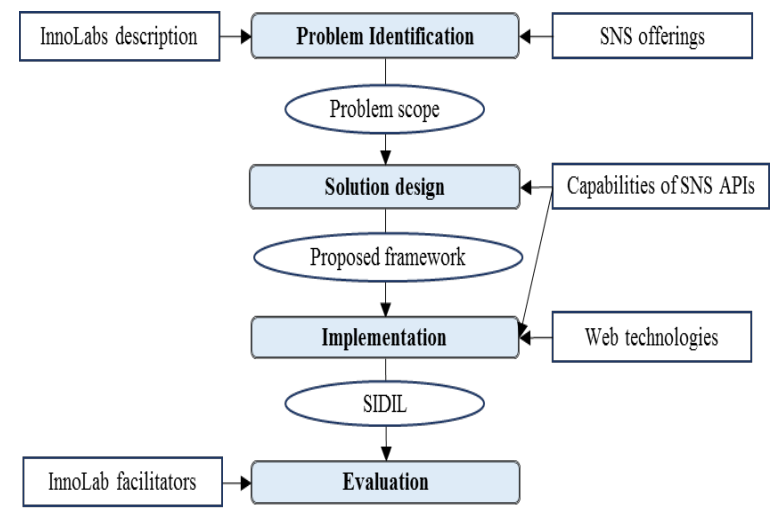

Fig. 1: Overall research procedure of the study

The domain-specific attributes that frame the distinct description of InnoLabs that need to be represented on online platforms was mainly adopted from [9]. Subsequently, a conceptual analysis of the offerings of leading extant SNSs (i.e. Facebook, LinkedIn, Google+, and Xing) was undertaken to reveal their respective strengths and shortcomings in supporting the web representation of InnoLabs in terms of their general as well as domain-specific attributes. The feature list of online representation being supported by these SNSs aggregately was retrieved from [10] and then support from individual networks for the representation of these identified attributes was measured by visiting several existing business pages and also creating sample business pages on each of the included SNSs. As a result, the objectives and the conceptual framework of new artifact was solicited at the end of this phase. In the second phase, the architecture and a working model of proposed solution were designed and implemented for the case domain - InnoLabs. The designed artifact is called SIDIL - Social networks Integrated Directory of Innovation Laboratories. The efficacy and performance of SIDL was iteratively tested between the designing and evaluation stages of its on-going implementation. Then, at the end of development process, the effectiveness and usability of SIDIL was evaluated by InnoLab facilitators (the targeted user community of the system). In doing so, the SIDIL prototype was introduced to the evaluators and subsequently they were asked for evaluation by filling out the evaluation questionnaire.

\section{Problems in Investigation \& Proposed De- sign}

The problem that this research aims to address was explored in more detail by examining the requirements of web representation of a special kind of organizations, called Innovation Laboratories (hereafter referred to 
as InnoLabs). InnoLabs are defined as the dedicated and specialized physical spaces that help the organizations in developing the new or improving their existing innovative products and services through the provision of expert advice and facilitation and the required innovation resources and mediating services $[9,11]$. They can be regarded as business organizations in a way that they are standalone and work for their customers (other business organizations). However, they are different from usual business organizations as there is no selling product at their disposal rather a different set of intangible innovation services. Thus, they are characterized in a different style than the usual manufacturing styled business organizations.

According to the recent studies on the phenomenon of InnoLabs (e.g. [12], [9], [13], [14]), it is evident that the term InnoLab in the practice is being applied to different constructs and thus they can be differentiated in a number of ways. They can be characterized on the basis of the innovation object they help to innovate, innovation services they provide, innovation resources they have, part of innovation process they support, types of innovation they focus, thematic concern, geographic orientation, business model, management structure, and the like. Furthermore, based on different services and resources that they offer, they are categorized in different categories. The varying attributes of InnoLabs serve to differentiate them from one another within and across categories [9]. Accordingly, the representation of InnoLabs on a social network calls for representation of two types of characteristics; general features, and domain specific features. According to [9], there are 11 different domain-specific aspects that need to be defined with web-representation of InnoLabs in order to discover their actual competencies and be able to locate, distinguish, and select their offered services. Table 1 shows the list of general and domain specific attributes of InnoLabs that need to be defined and the support for their representation across four leading social networking sites. The general features list included herein is retrieved from business page architecture description given in [10]. The availability of support for representation of individual features is revealed by surveying several business pages hosted on existing SNSs and also by creating sample business pages on each platform.

As shown in Table 1, the social networks do support the representation of general attributes, however, the support for domain specific attributes is scarce. This makes it challenging for an InnoLab and also any other special type of organization to have complete web representation. Furthermore, as the representation on social networks is beneficial in reaching out and

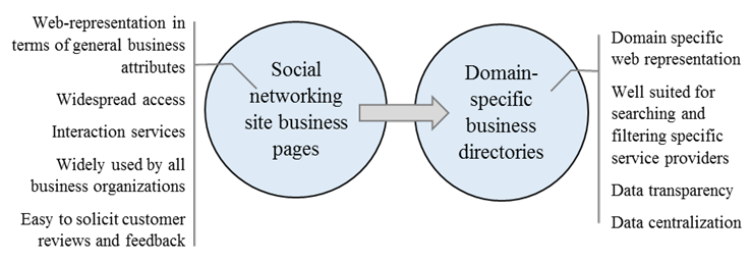

Fig. 2: Proposed approach for designing social networks integrated domain-specific business directories

interacting with user community; a lot of information is already being shared by these organizations on such networks. While there might be available some domain specific platforms dedicated for specific type of organizations, it becomes difficult for them to share useful information on all isolated platforms, and be able to collect, integrate, and utilize their dispersed social data and interactions. In this realm, we hereby propose to design domain-specific business directories for such special kind of organizations while simultaneously linking them to existing social networking sites (cf. Figure 2). Doing this will enable the domain specific applications to support web representation in terms of general as well as domain specific attributes without burdening the organizations to provide same information again. This will also help to aggregate and centralize business information that is available on different social networking sites.

\section{Implementation \& Evaluation}

The approach proposed herein is case implemented to design a domain specific directory of InnoLabs named as 'Social networks Integrated Directory of InnoLabs (abr. SIDIL)'. The application facilitates a digital directory and lookup facility for the InnoLabs in terms of their general (retrieved from social networks) as well as do-main specific attributes, and meanwhile aggregates and centralizes their available SNS information. Another feature of the SIDIL is to enable the discovery of homogeneous (offering similar services) and heterogeneous (offering different services) InnoLabs.

\subsection{User Interface}

The initial user interface of SIDIL presents a menu consisting of eight items.

- Loading a complete directory of registered InnoLabs

- Retrieving a list of InnoLabs that the user has previously bookmarked

- Retrieving a list of InnoLabs that the user has previously added to their network 


\begin{tabular}{|c|c|c|c|c|}
\hline Attribute & Facebook & LinkedIn & Google+ & Xing \\
\hline \multicolumn{5}{|c|}{ General InnoLab attributes } \\
\hline Name & $\checkmark$ & $\sqrt{ }$ & $\sqrt{ }$ & $\sqrt{ }$ \\
\hline Business logo & $\sqrt{ }$ & $\sqrt{ }$ & $\sqrt{ }$ & $\sqrt{ }$ \\
\hline Foundation year & $\checkmark$ & $\checkmark$ & $x$ & $\checkmark$ \\
\hline Business size & $x$ & $\sqrt{ }$ & $x$ & $\sqrt{ }$ \\
\hline Description & $\sqrt{ }$ & $\sqrt{ }$ & $\sqrt{ }$ & $\sqrt{ }$ \\
\hline Categorization & $\checkmark$ & $\checkmark$ & $\checkmark$ & $\checkmark$ \\
\hline Products & $\sqrt{ }$ & $\sqrt{ }$ & $x$ & $x$ \\
\hline Specialties & $\sqrt{ }$ & $\sqrt{ }$ & $x$ & $x$ \\
\hline Website & $\checkmark$ & $\checkmark$ & $\checkmark$ & $\checkmark$ \\
\hline Address & $\checkmark$ & $\checkmark$ & $\checkmark$ & $\checkmark$ \\
\hline Phone No. & $\checkmark$ & $\times$ & $\sqrt{ }$ & $\sqrt{ }$ \\
\hline Email & $\sqrt{ }$ & $x$ & $\sqrt{ }$ & $\sqrt{ }$ \\
\hline Fax & $x$ & $x$ & $x$ & $\sqrt{ }$ \\
\hline \multicolumn{5}{|c|}{ Domain-specific InnoLab attributes } \\
\hline InnoLab category & $\times$ & $\times$ & $x$ & $x$ \\
\hline Innovation object & $\times$ & $x$ & $\times$ & $x$ \\
\hline Innovation process & $\times$ & $\times$ & $\times$ & $\times$ \\
\hline Innovation resources & $\times$ & $\times$ & $\times$ & $\times$ \\
\hline Innovation focus & $\times$ & $x$ & $x$ & $\times$ \\
\hline Innovation methodology & $x$ & $x$ & $x$ & $x$ \\
\hline Business model & $x$ & $x$ & $\times$ & $x$ \\
\hline Innovation scope & $\times$ & $x$ & $\times$ & $\times$ \\
\hline Regional orientation & $x$ & $\times$ & $x$ & $x$ \\
\hline Thematic orientation & $x$ & $x$ & $x$ & $x$ \\
\hline Management structure & $x$ & $x$ & $x$ & $x$ \\
\hline
\end{tabular}

TABLE 1: InnoLab general and domain-specific attributes and support for their representation across existing SNSs

- Searching through keywords with a possible restriction to a particular field

- Searching through advanced filtering of data fields

- Navigational search

- Searching for homogeneous InnoLabs

- Searching for heterogeneous InnoLabs.

The searching for homogeneous and heterogeneous InnoLabs is made with respect to the profile of loggedin user in the case that the user is an InnoLab itself. The user defines one or more attributes as matching criteria and also indicates whether matching of a single or all values for a multi-valued data field is required for qualifying for similarity or dissimilarity, respectively.

Upon the execution of search request on the server side, the user interface presents the list of retrieved InnoLabs along with their integrated geographic map, faceted filters (flat and multi-valued), and details of the first InnoLab of the list. When the user selects a particular InnoLab, the system displays the detailed profile of select-ed InnoLab inside the 'InnoLab details' section together with an action pane (on the top). The action pane allows to add the selected InnoLab in the user's bookmark list, user's network list, interact with InnoLab in real-time through Skype, and access the Facebook, LinkedIn, Google+, and Xing page of the selected InnoLab. Subsequently, upon the selection of

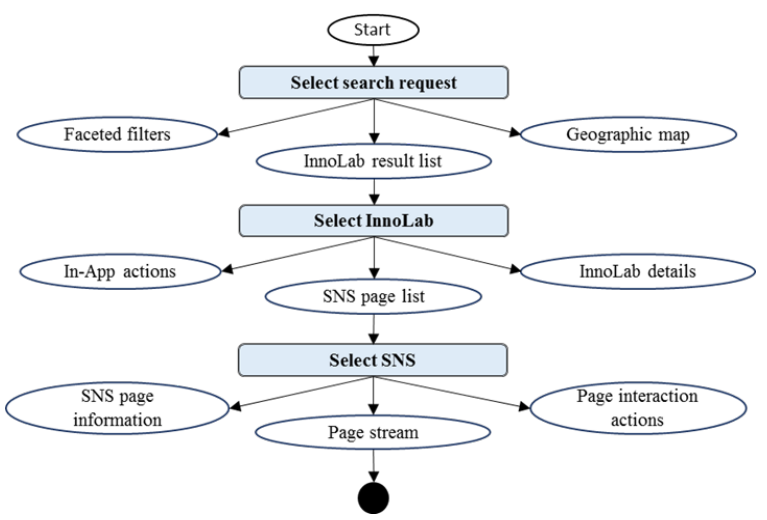

Fig. 3: A simplified view of SIDIL-User interaction workflow

a particular SNS, the system displays InnoLab information retrieved from selected SNS page together with a sub action pane. Sub action pane enables to retrieve page stream, like or follow the page, and interact with the page. A simplified view of SIDIL-User interaction workflow is shown in Figure 3 and a snapshot of SIDIL user interface showing the directory of InnoLabs is given in Figure 4. 


\subsection{Technical Implementation}

The SIDIL prototype is constructed and configured in a typical three-tier application style upon WAMP platform. The application is developed in PHP (serverside), and HTML and JavaScript with jQuery library ${ }^{1}$ (client-side) using a relational MySQL database. Asynchro-nous JavaScript calls with JavaScript Object Notation are used for generating dynamic content with respect to user's request asynchronously. For the client side sort-ing and filtering of retrieved data, application uses Ja-vaScript Underscore library ${ }^{2}$ and Google Maps JavaS-cript $\mathrm{API}^{3}$ is used for generating the integrated geo-graphical map of retrieved InnoLab locations. The local database is accessed with SQL commands in PHP scripts. The application leverages the services of appropriate social networking APIs ${ }^{4567}$ for interconnecting with remote data stores of SNSs. In order to accomplish the semantic and natural language related functionalities, the application uses PHP based phpMorphy li-brary ${ }^{8}$ and corpus-based web services offered by Leipzig Corpora Collection ${ }^{9}$ using its REST $\mathrm{API}^{10}$.

The overall search and data fetching functionality on the server side of SIDIL can be decomposed into several tasks. The kind of fetch request committed by user determines the execution of particular tasks (cf. Figure 5). In this regard, the server initially prepares the semantic search request according to a semantic search procedure similar to the semantic tokenization scheme employed in [15]. Successively, the server performs a full-text search over complete (basic and the InnoLab specif-ic) profile of all SIDIL members while iterating through the list of search terms. In order to filter the search results for relevant InnoLabs with respect to user request, the similarity of data fields is determined according to the text matching approach proposed in [15]. The identification of and the interconnection with SNS business pages of selected InnoLab is achieved by the combination of the page source (i.e. name of the SNS platform) and the page ID which is retrieved at the time of connecting the particular page. The data from correspond-ing page is retrieved

1. https://jquery.com

2. http://underscorejs.org

3. https://developers.google.com/maps/documentation/javascr

4. https://developers.facebook.com/docs/graph-api

5. https://docs.microsoft.com/en-us/linkedin

6. https://developers.google.com

7. https://dev.xing.com

8. http://phpmorphy.sourceforge.net/dokuwiki

9. http://corpora.uni-leipzig.de

10. http://wortschatzwebservices.informatik.unileipzig.de/ws/swagger-ui.html

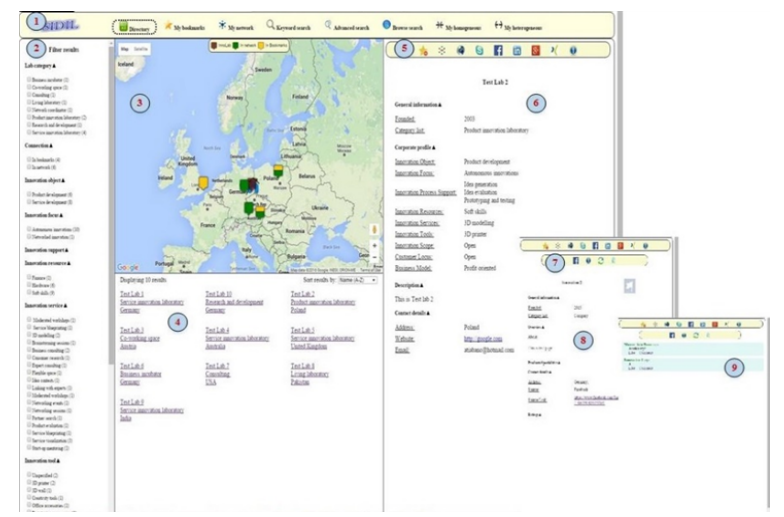

Fig. 4: An aggregated screenshot of SIDIL user interface. (1) Main menu of SIDIL, (2) Faceted filters, (3) Geographic map, (4) List of retrieved InnoLabs, (5) Detailed profile of the selected InnoLab, (6) Action pane, (7) SNS page action pane, (8) SNS page information

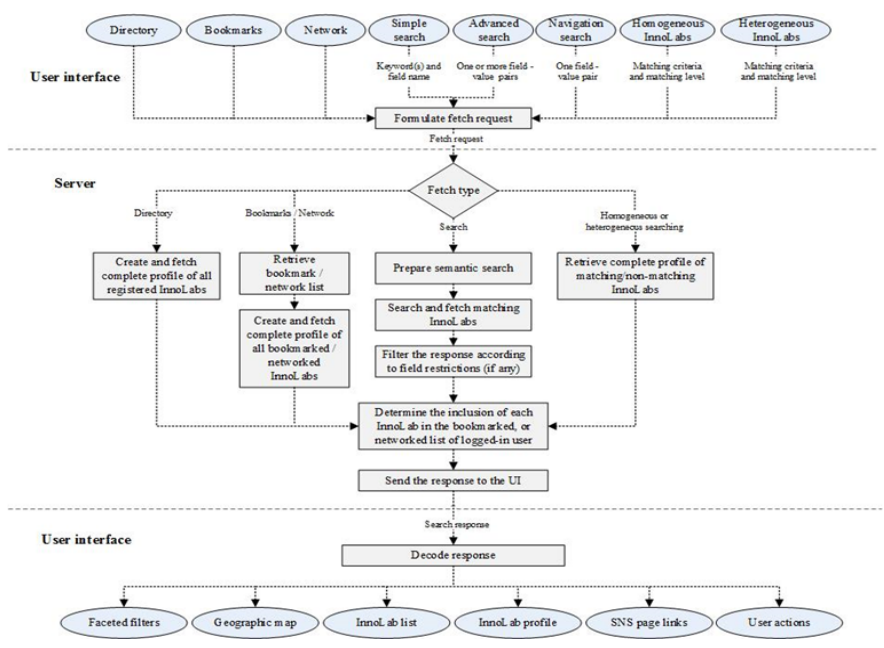

Fig. 5: The fetch procedure of the SIDIL

and successively mapped to the native data format in order to support uniform and consistent information view across different social networking platforms.

\subsection{Evaluation}

The SIDIL application has been tested and validated by integrating it into prototype of a larger InnoLab collaboration platform that supports inter-connectivity among InnoLabs throughout the collaboration proresss. The prototype has been evaluated by three InnoLab facilita-tors (experts running an InnoLab). The evaluators were selected from different regions including one evaluator form USA who is the head of an InnoLab that mainly focuses on business incubation services, and two evaluators from Europe who are heads of InnoLabs that mainly offer a living lab environment for innovation processes. 


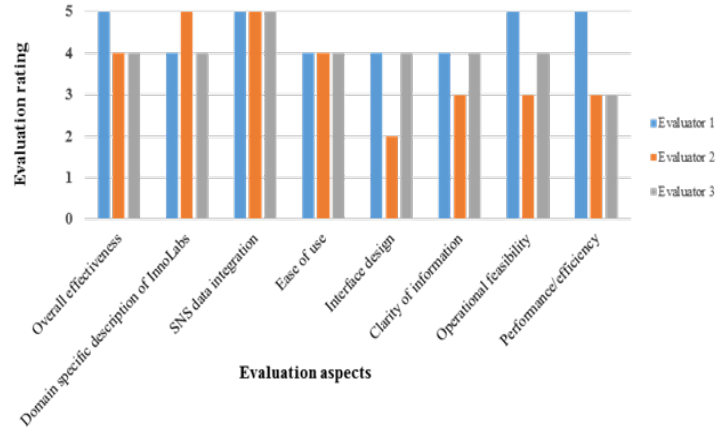

Fig. 6: Item-wise evaluation responses of SIDIL $(\mathrm{N}=3)$

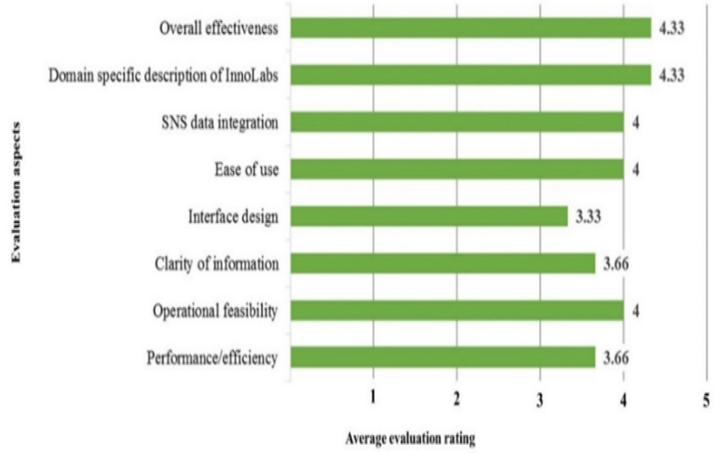

Fig. 7: Aggregated evaluation results of SIDIL ( $\mathrm{N}=3$ )

The evaluators were first presented with the application in isolated mode and a walk-through of the application was given. Having understood the application objectives and functionality, the evaluators checked the prototype and successively ranked its effectiveness and usability in achieving the targeted goals by responding to a questionnaire. The evaluation questionnaire included 8 items related to the SIDIL effectiveness in achieving its targeted goals. The responses were record-ed on a Likert type scale ranging from 1 (lower - most negative) to 5 (higher - most positive). The evaluation data was fed into the excel sheet and a descriptive analysis of data was performed. The results were aggregated item-wise and an average score for each item was computed and relative charts were drawn. Figure 6 shows individual evaluation responses of all evaluators to each evaluation item; while the aggregated item-wise results of the evaluation of SIDIL are shown in Figure 7. As shown, the application was found effective (score higher than 4 on evaluation items 1-3) in supporting the domain specific description of InnoLabs and their SNS data integration. Similarly, the application was also found usable and efficient (score higher than 3 on evaluation items 4-8).

\section{References}

[1] Rysman M, "Competition between networks: A study of the market for yellow pages." The Review of Economic Studies, Vol. 71, pp. 483-512, 2004.

[2] Ellison NB, "Social network sites: Definition, history, and scholarship." Journal of computer-mediated Communication, Vol. 13, pp. 210-230, 2007.

[3] Jefferson III CE, Traughber S, "Social media in business. How Social Media Can Help Small Businesses and NonProfit Organizations", pp. 2-3, 2012.

[4] Memon AB, Meyer K, "Why We Need Dedicated Webbased Collaboration Platforms for Inter-organizational Connectivity? A Research Synthesis." International Journal of In-formation Technology and Computer Science, Vol. 9, pp. 1-11, 2017.

[5] Hevner AR, March ST, Park J et. al., "Design science in information systems research." MIS quarterly, pp. 75-105, 2004.

[6] Takeda H, Veerkamp P, Yoshikawa H, "Modeling de-sign process." AI magazine, Vol. 11, 1990.

[7] Peffers K, Tuunanen T, Rothenberger MA et. al., "A design science research methodology for information systems research." Journal of management information systems, Vol. 24, pp. 45-77, 2007.

[8] Wieringa R, "Design science as nested problem solving." In Proceedings of the 4th international conference on design science research in information systems and technology, pp 1-12, 2009.

[9] Memon AB, Meyer K, Thieme M et. al., "Inter-InnoLab collaboration: An investigation of the diversity and interconnection among Innovation Laboratories." Journal of Engineering and Technology Management, Vol. 47, pp. 1-21, 2018.

[10] Memon AB, Meyer K, "Affordances of Business Pages on Social Networking Sites: Towards an Integration Model." International Journal of Social Media and Online Communities, Vol. 12, 2020.

[11] Memon AB, Meyer K, "Towards the Functional Roles of an Innovation Laboratory as a Platform for Innovation: An Observational Approach." International Journal of Service Science, Management, Engineering, and Technology (IJSSMET), Vol. 8, pp. 32-49, 2017.

[12] Memon AB, Meyer L-P, Meyer K et. al., "An Insight Survey of Innovation Laboratories Worldwide." Advances in The Human Side of Service Engineering, Vol. 1, pp. 174-185, 2014.

[13] Schmidt J, "Erarbeitung und Anwendung eines strukturierten Kriterienkatalogs zum Vergleich von Innovationslaboren." Seminararbeit Universität Leipzig, 2009.

[14] Puttick, Ruth. "Innovation Teams and Labs: A practice guide."London: NESTA. http://www.nesta. org.uk/publications/innovation-teams-and-labs-practiceguide. Accessed October 12 (2014): 2016.

[15] Memon AB, Zinke C, Meyer K, "A Semantics-Based Approach for Business Categorization on Social Networking Sites." In Working Conference on Virtual Enterprises, pp 678-687, 2017. 